\title{
YouTube as an information source during the COVID-19 outbreak: a cross sectional study of Turkish video content
}

\author{
DSerhat Sirekbasan'1, Serap Süzük Yıldız ${ }^{2}$ \\ ${ }^{1}$ Çankırı Karatekin University, Eldivan Vocational School of Health Services, Department of Medical Services and Techniques, Çankırı, Turkey \\ ${ }^{2}$ Ministry of Health General Directorate of Public Health, Department of Microbiology Reference Laboratory and Biological Products, \\ Ankara, Turkey
}

Cite this article as: Sirekbasan S, Süzük Ylldız S. YouTube as an information source during the COVID-19 outbreak: a cross sectional study of Turkish video content. J Health Sci Med 2021; 4(3): 340-343.

\begin{abstract}
Aim: Although videos posted on YouTube are popular sources of information on public health issues, they generally need confirmation. Our aim in this study was to evaluate the content of the most viewed Turkish YouTube videos as an information source during the COVID-19 outbreak.

Material and Method: On March 26, 2021, videos containing information about the disease were searched on YouTube using the keywords 'coronavirus' and 'COVID-19'. The videos were classified as useful, misleading, personal experiences and news updates based on the type of information they contain. Inter-rater agreement was evaluated using the kappa coefficient. The total number of views, days since upload, total video time and upload source were noted.

Results: 96 of a total of 200 videos met the inclusion criteria. The total number of views of the 96 videos included was $21,598,563$. $47.92 \%$ of the videos were classified as useful, $37.5 \%$ as news updates, and $6.25 \%$ as personal experiences. It was determined that $8.33 \%$ of the videos presented medically misleading information.

Conclusion: This study shows that most of the internet videos about COVID-19 on YouTube are considered as beneficial. Videos prepared by reliable sources such as academic institutions and health institutions were few. It is very important that news agencies take measures to prevent the dissemination of false information in public health emergencies and that the content of the videos they publish is correct.
\end{abstract}

Keywords: Knowledge, COVID-19, epidemic diseases, YouTube, public health

\section{INTRODUCTION}

The novel coronavirus (COVID-19) emerged in Wuhan, China in December 2019, spread rapidly in a short time, and affected the whole world (1). The World Health Organization (WHO) declared the COVID-19 outbreak a pandemic on March 11, 2020 (2). As of September 20, 2020 , it became undoubtedly one of the fatal pandemics in history, with more than 30 million reported cases and 954417 deaths globally (3). The most critical intervention to control the spread of COVID-19 infection is to implement preventive measures. These measures generally include identifying, treating, and isolating infected individuals (4). Moreover, it is also particularly essential to educate the public about the disease, convey correct information to all communities, and prevent fake news and misinformation.
With an unprecedented increase in internet use, online platforms have become digital settings where healthrelated information can be easily accessed. YouTube, a popular video-sharing platform, is one of the most dominant online information sources, with over a billion users generating billions of views daily (5). It has the potential to be a unique tool for the timely sharing and dissemination of accurate information on a large number of medical conditions. However, video content without helpful information can be a misleading information source against positive efforts to prevent infection, especially during pandemics (6). Ultimately, we aimed to examine the accuracy, usability, and effective use of the most viewed YouTube videos with Turkish content related to COVID-19. 


\section{MATERIAL AND METHOD}

Since our study was digital survey research conducted with the help of YouTube videos available on the web browsers and open to everyone, it did not require any ethical approvals.

\section{Search Protocol}

On March 26, 2021, we searched for videos with diseaserelated content on YouTube (http://www.youtube.com) using the keywords 'coronavirus' and 'COVID-19.' As the web browser, we utilized the latest version of Google Chrome with all available updates. We viewed the videos through a cleared cache by deleting the respective browser's cookies, personal preferences, and browsing history data.

We limited our search to the top 100 videos for each keyword and used the YouTube algorithm as the criteria for sorting the videos by the "relevance" filter.

We saved a total of 200 search results filtered by keywords in a playlist, as search results on YouTube can change every day and will likely yield different results during the pandemic. We extracted the URLs of the videos listed for each keyword to a spreadsheet.

\section{Inclusion and Exclusion Criteria}

Exclusion criteria included non-Turkish videos, reproduced videos, videos without audio-visual information, videos longer than 15 minutes, and live broadcasts. Besides, we also excluded the ones without medical content related to COVID-19 (e.g., political aspects of the outbreak, the impact of the disease on the economy, etc.).

On the other hand, we included key identifying attributes of all videos, including video title, number of views, likes and dislikes, video upload date, and video length.

\section{Evaluation of Videos}

The researchers reviewed and analyzed all the videos independently. Based on the information and reviews provided, we classified the videos as useful, misleading, personal experiences, and news updates. Useful videos were defined as those containing scientifically correct information about any aspect of the disease (epidemiology, transmission, symptoms, diagnostic tests, treatment, prevention). Misleading videos included content providing at least one scientifically unproven piece of information (e.g., COVID-19 is an artificial conspiracy or a population reduction strategy, etc.) or vague claims that cannot be evidencebased. We defined videos with personal experience as those providing content related to individuals' own experiences or the experiences of their family members, relatives, friends, or neighbors suffering from COVID-19. Finally, news updates were the videos uploaded by news agencies, healthcare organizations, or independent users and lacked helpful information about COVID-19 and contained information about the current state of the disease, such as mortality.

We were aware of the uncertainties regarding COVID-19. For example, there was no consensus on the source of the pandemic, the contagiousness of the virus, the impacts of the measures, the exact incubation period of the virus, the severity of the disease, and mortality rates. Therefore, before evaluating the videos, we reviewed the COVID-19related information on the WHO website and accepted its content as standard and scientifically up-to-date.

\section{Statistical Analyses}

We run statistical analyses with IBM SPSS 20.0 (IBM Corp., Armonk, NY, USA) package program and Microsoft Excel version 2013. We determined frequency $(\mathrm{n})$, percentage (\%), median (Md), and mean (M) in the analysis of the collected data. We calculated Cohen's kappa coefficient $(\kappa)$ to value the interrater agreement. Finally, we used the Mann-Whitney $U$ test to compare the features of useful and misleading videos. A value of $p$ $<0.05$ was considered statistically significant.

\section{RESULTS}

We did a search using the specified keywords and viewed the first 200 videos for relevance by our selection criteria. Of the 200 videos viewed, 96 met the inclusion criteria. Nevertheless, we excluded 56 non-Turkish videos, 17 fully or partially reproduced videos, 12 videos longer than 15 minutes, and 16 irrelevant videos (Figure). Besides, we calculated Cohen's kappa coefficient as 0.93 , indicating a perfect interrater agreement.

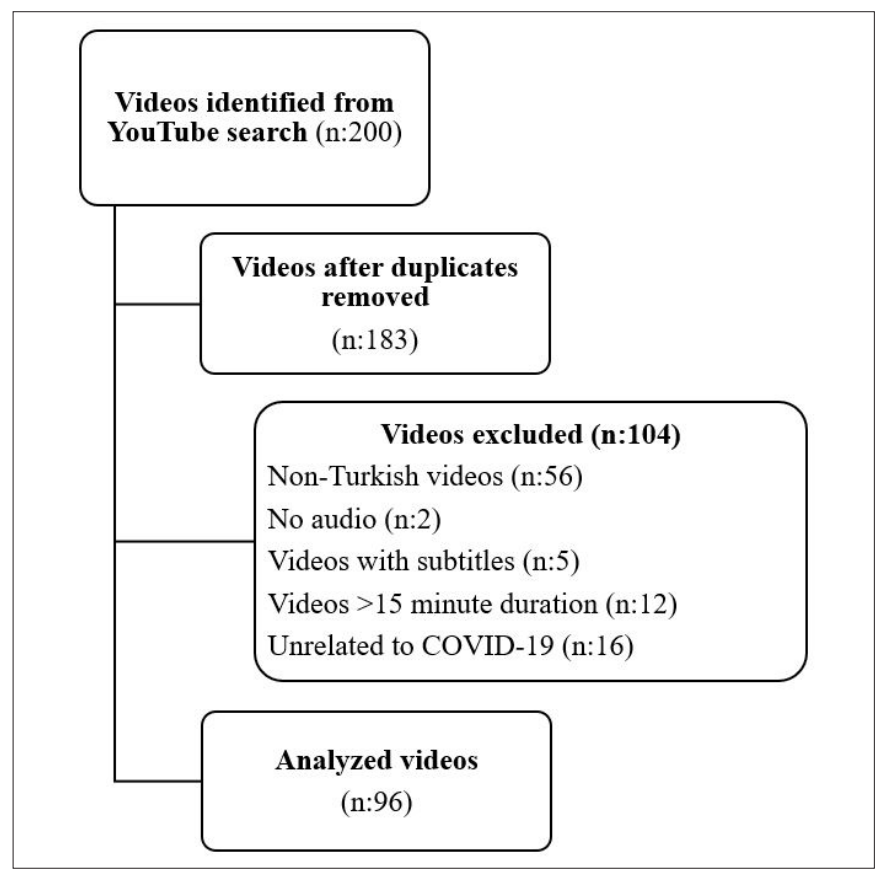

Figure. 
The total number of views of the 96 videos included was $21,598,563$. The total length of all the videos was 6.53 hours. These 96 videos reached a total of 187,514 likes. We found a significant difference between the lengths of useful and misleading videos $(\mathrm{p}<0.05$; Table).

\begin{tabular}{|c|c|c|c|}
\hline Video features & Useful $(n=46)$ & Misleading $(\mathrm{n}=8)$ & $\mathbf{P}$ \\
\hline $\begin{array}{l}\text { Number of } \\
\text { views }\end{array}$ & $\begin{array}{c}20900 \\
(196-2755249)\end{array}$ & $\begin{array}{c}101907 \\
(2426-895252)\end{array}$ & 0.319 \\
\hline $\begin{array}{l}\text { Number of } \\
\text { likes }^{*}\end{array}$ & $\begin{array}{c}273.5 \\
(0-46294)\end{array}$ & $\begin{array}{c}506 \\
(23-10084)\end{array}$ & 0.331 \\
\hline $\begin{array}{l}\text { Number of } \\
\text { dislikes }\end{array}$ & $\begin{array}{c}15 \\
(0-849)\end{array}$ & $\begin{array}{c}62 \\
(4-729)\end{array}$ & 0.214 \\
\hline $\begin{array}{l}\text { Video lengths } \\
\text { (minute) }^{*}\end{array}$ & $\begin{array}{c}4.23 \\
(0.37-15)\end{array}$ & $\begin{array}{c}1.43 \\
(1.12-3.5)\end{array}$ & $0.010^{\star *}$ \\
\hline
\end{tabular}

Overall, we classified 46 (47.92\%) videos as useful, $36(37.5 \%)$ as news updates, $6(6.25 \%)$ as personal experiences, and $8(8.33 \%)$ as misleading.

Most of the videos were uploaded by news agencies (73.96\%; n:71) and healthcare organizations (12.5\%; $\mathrm{n}: 12)$. The Ministry of Health contributed only one video to this series. The number of videos uploaded by individual users was 10 (10.42\%). Finally, we determined the number of videos shared by academic institutions to be $3(3.13 \%)$.

\section{DISCUSSION}

The whole world has been struggling with COVID-19 caused by severe acute respiratory syndrome coronavirus 2 (SARS CoV-2) since December 2019 (7). We believe that the focus of further studies in tackling this pandemic will be the development of effective medicines and vaccines to treat COVID-19. Nevertheless, the most important task for us in the near future should be the adequate communication of accurate disease-related information among the public. Therefore, we aimed to examine the accuracy of the most viewed COVID-19-related YouTube videos with Turkish content as information sources. We believe that the data in this study will provide a perspective on the reliability of information sources on efforts to control the spread of the disease.

Our study qualified most of the YouTube videos about COVID-19 as useful. Considering the mean number of views, popularity was similar between useful and misleading videos. These findings are consistent with studies that previously evaluated YouTube's role as a medical information source on H1N1 influenza (8), Ebola virus outbreak (9), West Nile virus (10), Zika virus outbreak (11), and vaccination (12).
Previous studies reported that approximately 15 to $30 \%$ of information on YouTube about any disease could be misleading (13-15). Misinformation about COVID-19 is widespread on the internet and social media. In their study analyzing the quality of online information on COVID-19, Cuan-Baltazar et al. compared the information on 36 websites with the relevant medical literature (PubMed) and found that 15 websites provided accurate information, 16 provided partially accurate information, and 5 reported misleading information (16). Aiming at determining the size of misleading information spread on Twitter (Twitter, Inc., San Francisco, CA), Kouzy et al. (17) reviewed 673 tweets related to the COVID-19 outbreak and found the misinformation rate among the tweets as $24.8 \%$. Turkish videos available on YouTube were primarily informative. Among the 96 videos included, we found only $8(8.33 \%)$ videos to be misleading. These results are very pleasing and reassuring. The low rate of misleading videos in our study can be explained by the source of these videos being different compared to previous studies and the satisfying awareness around the world regarding the total fight against the destructive impact of COVID-19.

Akyol et al. (18) assessed videos about sarcopenia and reported that the videos uploaded by physicians and academic organizations had the highest quality. Similarly, the previous studies revealed that videos uploaded by academics and physicians had highest quality (19, 20). News agencies contributed to approximately $74 \%$ of the total videos, which was followed by healthcare institutions (12.5\%) and individual users (10.42\%), respectively. Given the global and rapid spread of the pandemic, it is expected for news agencies to attract more attention. Whereas the videos uploaded by individual users are more likely to contain misleading information than news agency videos, it was pretty remarkable that 8 misleading videos in this study were uploaded by news agencies. We believe that uncertainties in the early stages of the outbreak may have influenced the content of these videos.

\section{CONCLUSION}

This study revealed that the most viewed YouTube videos with Turkish content during the COVID-19 outbreak had missing information. One of the basic elements of combating this disease is that people know what to do to prevent it. Given this situation, the presence of videos containing fake information is an alarming issue. As a result, it can be argued that the videos uploaded to YouTube do not have editorial processes or do not intend to inform the public. Nonetheless, news agencies should mind reviewing the content and censoring misleading information in their news and videos they upload. 


\section{ETHICAL DECLARATIONS}

Ethics Committee Approval: Since our study was digital survey research conducted with the help of YouTube videos available on the web browsers and open to everyone, it did not require any ethical approvals

Referee Evaluation Process: Externally peer-reviewed.

Conflict of Interest Statement: The authors have no conflicts of interest to declare.

Financial Disclosure: The authors declared that this study had received no financial support.

Author Contributions: All of the authors declare that they have all participated in the design, execution, and analysis of the paper and approved the final version.

\section{REFERENCES}

1. Baloch S, Baloch MA, Zheng T, Pei X. The coronavirus disease 2019 (COVID-19) pandemic. Tohoku J Exp Med 2020; 250: 271-8.

2. World Health Organization. Rolling updates on coronavirus disease (COVID-19). https://www.who.int/emergencies/ diseases/novel-coronavirus-2019/events-as-they-happen?utm_ medium=email\&utm_source=transaction

3. World Health Organization. Coronavirus disease (COVID-19) pandemic. https://www.who.int/emergencies/diseases/novelcoronavirus-2019.

4. Sun P, Lu X, Xu C, Sun W, Pan B. Understanding of COVID-19 based on current evidence. J Med Virol 2020; 92: 548-51.

5. YouTube. YouTube Social impact. pandemic. https://socialimpact. youtube.com/.

6. Madathil KC, Rivera-Rodriguez AJ, Greenstein JS, Gramopadhye AK. Healthcare information on YouTube: a systematic review. Health Informatics J 2015; 21: 173-94.

7. Li H, Liu SM, Yu XH, Tang SL, Tang CK. Coronavirus disease 2019 (COVID-19): current status and future perspectives. Int J Antimicrob Agents 2020; 55: 105951.

8. Pandey A, Patni N, Singh M, Sood A, Singh G. YouTube as a source of information on the H1N1 influenza pandemic. Am J Prev Med 2010; 38: e1-3.

9. Pathak R, Poudel DR, Karmacharya P, et al. YouTube as a source of information on Ebola virus disease. N Am J Med Sci 2015; 7: 306-9.

10. Dubey D, Amritphale A, Sawhney A, Dubey D, Srivastav N. Analysis of YouTube as a source of information for West Nile virus infection. Clin Med Res 2014; 12: 129-32.

11. Bora K, Das D, Barman B, Borah P. Are internet videos useful sources of information during global public health emergencies? A case study of YouTube videos during the 2015-16 Zika virus pandemic. Pathog Glob Health 2018; 112: 320-28.

12. Keelan J, Pavri-Garcia V, Tomlinson G, Wilson K. YouTube as a source of information on immunization: a content analysis. JAMA 2007; 298: 2482-4

13. Kumar N, Pandey A, Venkatraman A, Garg N. Are video sharing web sites a useful source of information on hypertension? J Am Soc Hypertens 2014; 8: 481-90.

14. Delli K, Livas C, Vissink A, Spijkervet FK. Is YouTube useful as a source of information for Sjögren's syndrome? Oral Dis 2016; 22 196-201.

15. Singh AG, Singh S, Singh PP. YouTube for information on rheumatoid arthritis-a wakeup call? J Rheumatol 2012; 39: 899-903.

16. Cuan-Baltazar JY, Muñoz-Perez MJ, Robledo-Vega C, PérezZepeda MF, Soto-Vega E. Misinformation of COVID-19 on the Internet: Infodemiology Study. JMIR Public Health Surveill 2020; 6: e18444.
17. Kouzy R, Abi Jaoude J, Kraitem A, et al. Coronavirus goes viral: quantifying the COVID-19 misinformation epidemic on Twitter. Cureus 2020; 12: e7255.

18. Akyol A, Karahan İ. Is YouTube a quality source of information on sarcopenia? Eur Geriatr Med 2020; 11: 693-7.

19. Kocyigit BF, Akaltun MS. Does YouTube provide high quality information? Assessment of secukinumab videos. Rheumatol Int. 2019; 39: 1263-8.

20. Tolu S, Yurdakul OV, Basaran B, Rezvani A. English-language videos on YouTube as a source of information on self-administer subcutaneous anti-tumour necrosis factor agent injections. Rheumatol Int. 2018; 38: 1285-92. 